\title{
A Network Analysis of DSM-5 posttraumatic stress disorder symptoms and correlates in U.S. military veterans.
}

\author{
Cherie Armour*, Ph.D. ${ }^{a} \&$ Eiko I. Fried*, Ph.D. ${ }^{\mathrm{b}}$ \\ Marie K. Deserno ${ }^{\mathrm{c}}$ \\ Jack Tsai, Ph.D. ${ }^{\text {d,e }}$ \\ Robert H. Pietrzak, Ph.D., M.P.H. ${ }^{\mathrm{d}, \mathrm{f}}$
}

*These authors contributed equally to the manuscript

a Psychology Research Institute, University of Ulster, Coleraine, Northern Ireland, UK

${ }^{\mathrm{b}}$ Faculty of Psychology and Educational Sciences, University of Leuven, Leuven, Belgium

${ }^{c}$ Department of Psychology, University of Amsterdam, Amsterdam, The Netherlands

${ }^{\mathrm{d}}$ Department of Psychiatry, Yale University School of Medicine, New Haven, CT, USA

${ }^{\mathrm{e}}$ United States Department of Veterans Affairs, New England Mental Illness Research, Education, and Clinical Center, West Haven, CT, USA

${ }^{\mathrm{f}}$ United States Department of Veterans Affairs, National Center for Posttraumatic Stress

Disorder, Clinical Neurosciences Division, West Haven, CT, USA

*Correspondence: Cherie Armour: Psychology Research Institute, Coleraine Campus, Ulster University, Coleraine, Northern Ireland, UK, BT52 1SA

Tel: (0044) 02870123374

Email: armour.cherie@gmail.com 


\begin{abstract}
Object: Recent developments in psychometrics enable the application of network models to analyze psychological disorders, such as PTSD. Instead of understanding symptoms as indicators of an underlying common cause, this approach suggests symptoms co-occur in syndromes due to causal interactions. The current study has two goals: (1) examine the network structure among the 20 DSM-5 PTSD symptoms, and (2) incorporate clinically relevant variables to the network to investigate whether PTSD symptoms exhibit differential relationships with suicidal ideation, depression, anxiety, physical quality of life (QoL), mental QoL, age, and sex. Method: We utilized a nationally representative U.S. military veteran's sample; and analyzed the data from a subsample of 221 veterans who reported clinically significant DSM-5 PTSD symptoms. Networks were estimated using Gaussian graphical models and the lasso regularization. Results: The 20-item DSM-5 PTSD network revealed that symptoms were positively connected within the network. The most central symptoms were negative trauma-related emotions, flashbacks, detachment, and physiological cue reactivity. Especially strong connections emerged between nightmares and flashbacks; blame of self or others and negative trauma-related emotions, detachment and restricted affect; and hypervigilance and exaggerated startle response. Incorporation of clinically relevant covariates into the network revealed paths between self-destructive behavior and suicidal ideation; concentration difficulties and anxiety, depression, and mental QoL; and depression and restricted affect. Conclusion: These results demonstrate the utility of a network approach in modeling the structure of DSM-5 PTSD symptoms, and suggest differential associations between specific DSM5 PTSD symptoms and clinical outcomes in trauma survivors. Implications of these results for informing the assessment and treatment of this disorder, are discussed.
\end{abstract}

Keywords: PTSD; Depression; DSM-5; Veterans; Network Analysis; Functioning; Quality of Life (QoL); Suicide 


\section{Introduction}

The nosology of posttraumatic stress disorder (PTSD) is highly controversial and widely debated (McNally, 2004; Rosen, 2004), and there have been several changes to the diagnostic criteria since the initial introduction of PTSD into the Diagnostic and Statistical Manual of Mental Disorders (DSM). These changes include the definition of the criterion A trauma, the number and nature of symptoms (of note, DSM-5 now includes three new diagnostic symptoms of negative belief, distorted blame, and recklessness), the manner in which symptoms cluster in symptom groups, and the recent inclusion of a dissociative PTSD subtype. The latest version of the criteria (DSM-5) was released in May 2013. To date, however, relatively few studies have analyzed the DSM-5 criteria. The current study utilizes a novel psychometric approach based on Network Analysis to identify the way in which DSM-5 PTSD symptoms interact at the individual item level and in turn to identify if these interactions are clinically relevant.

PTSD is an impairing disorder that affects a significant proportion of both civilians and military personnel exposed to a traumatic event (Karam et al., 2014; Kessler et al., 2013; Pietrzak, Goldstein, Southwick, \& Grant, 2011). Lifetime estimates of PTSD in nationally representative samples reach $6.8 \%$ in the US (Kessler \& Üstün, 2008; Pietrzak et al., 2011), with estimated prevalence rates in military veteran populations ranging from 9-30\% (Lapierre, Schwegler, \& Labauve, 2007; Sundin et al., 2010; Tsai et al., 2014). Epidemiological studies suggest that interpersonal traumas such as assaultive violence have a higher conditional probability of PTSD symptom development than non-interpersonal traumas, such as unexpected loss of a loved one (Creamer, Burgess, \& McFarlane, 2001; Kessler et al., 1995). It is also reported that veterans displaying posttraumatic symptomatology have greater difficulty with re-integration to civilian life

post-deployment (Karstoft, Armour, Andersen, Bertelsen, \& Madsen, 2014); report a lower quality of life (QoL) (Giacco, Matanov, \& Priebe, 2013); and have greater risk for suicidal ideation (SI) 
(Jakupcak, Cook, Imel, Rosenheck \& McFall, 2009; Jakupcak et al., 2011). PTSD is additionally a highly comorbid disorder with the vast majority of individuals also meeting the criteria for at least one additional psychiatric disorder (Brady, Killeen, Brewerton, \& Lucerini, 2000; Pietrzak et al., 2011).

\subsection{PTSD as characterized by the DSM-5}

The most recent edition of the DSM (DSM-5; APA, 2013) characterizes PTSD as containing 20 individual symptoms that are grouped across four symptom clusters; Intrusions (B1B5; see Figure 1), Avoidance (C1-C2), Negative alterations in cognitions and mood (NACM; D1D7), and Alterations in arousal and reactivity (AAR; E1-E6). A diagnosis of PTSD requires that trauma survivors endorse a minimum of six symptoms ( 1 from the intrusions symptom cluster, 1 from avoidance, 2 from NACM, and 2 from AAR), in addition to reporting significant functional impairment and the persistence of symptoms in excess of one month (APA, 2013).

Notably, this prescribed six-item diagnostic algorithm is reported as being so amorphous in nature that it results in 636,120 combinations of PTSD symptomatology (Galatzer-Levy \& Bryant, 2013). Consequently, individuals with a DSM-5 PTSD diagnosis can have remarkably distinct symptom presentations. Another potential drawback of the current criteria is that individuals who fail to meet them (by exhibiting less than six symptoms in the prescribed DSM-5 fashion) may be as impaired by their symptomatology as individuals who do meet the criteria. For example, failing to endorse at least one avoidance item would preclude someone from the diagnosis even if all other items in all other symptom groups were endorsed.

\subsection{PTSD as a categorical or dimensional disorder}

Although the DSM acknowledges that psychiatric disorders are dimensional, its guidelines maintain that a threshold on the dimension is required in order to define who does vs. does not receive a diagnosis; the DSM-5 nosology for PTSD is thus embedded, from a diagnostic point of 
view, in a categorical framework. Contrasting this categorical approach, many researchers support a dimensional view to PTSD, stating that the distress experienced by an individual post-trauma exists on a continuum in which the high end reflects more severe distress (cf. Ruscio, Ruscio, \& Keane, 2002). In this case, symptoms that reflect PTSD are summed together to determine the position of an individual on the continuum. These sum scores are often used by researchers to assess the PTSD construct, particularly as it relates to the longitudinal course of symptoms (Bonanno et al., 2004; Dickstein, Suvak, \& Litz, 2010; Karstoft, Armour, Elklit, \& Solomon, 2013) and in assessing PTSD treatment response over time (Richardson et al., in Press).

\subsection{A network approach to PTSD}

Irrespective of whether the syndrome of PTSD is viewed through a categorical or dimensional lens, the commonality between these viewpoints is the proposition that symptoms are reflective indicators of an underlying latent construct that is PTSD. This 'reflective model' is the prevailing perspective with which psychopathology is currently viewed and understood (Borsboom, 2008; Borsboom \& Cramer, 2014). In both categorical and dimensional systems, PTSD symptoms are interchangeable and equally reflective of the latent construct that is PTSD; this view is known as the 'common cause hypothesis' (Fried, 2015; Schmittmann et al., 2013). A challenge is that symptom groupings as outlined in the DSM (detailed above) have been shown to vary in relation to the precipitating traumatic event (Armour \& Shevlin, 2010); vary in their comorbidity with alternative disorders (Contractor et al., 2014a,b; Pietrzak, Tsai, Armour, Mota, Harpaz-Rotem, \& Southwick, 2014; Roley et al., in press); relate differentially to perceived quality of life (Giacco et al., 2013); and display differential responses to treatment (Asmundson, Stapeleton \& Taylor, 2004). This implies that PTSD symptoms are not (roughly) interchangeable indicators of one underlying reflective latent variable that causes the covariation among symptoms. In addition, cross-sectional studies have revealed that different groups of individuals, displaying 
different symptom profiles, show differential associations with external variables (Ayer et al., 2011; Au et al., 2013; Breslau et al., 2005; Geronazzo Alman et al., 2012; Maguen et al., 2013; Naifeh et al., 2010; Rosellini et al., 2014).

Challenges to the common cause hypothesis extend past PTSD into the broader constellation of psychiatric disorders. Borsboom and Cramer (2014) highlighted that a disorder can only be the common cause of symptoms if they are locally independent, meaning that relations among symptoms are spurious and disappear once controlling for the common cause. That symptoms do not interact with each other causally, however, is highly implausible in psychopathology, as psychiatric symptoms by their very nature have direct relations to one another. This is exemplified by McNally et al. (2015), who showed that being presented with a reminder of a traumatic event may in turn trigger psychological and physiological reactions, which may activate avoidance behaviors; it is very likely that symptoms are directly related in dynamic causal webs of influences. This has important clinical implications, given that the ability to identify particular central symptoms in a causal system (symptoms that are highly connected and likely to trigger other symptoms), would allow clinicians to focus on such symptoms in assessment, monitoring, and treatment (Fried, Epskamp, Nesse, Tuerlinckx, \& Borsboom, 2015; Cramer et al., 2014). From the traditional perspective of reflective latent variables that cause the covariance among symptoms, such specific symptom-focused interventions would generally not be employed.

A new and growing school of thought in which symptoms are embedded within a causal network - and are causally related amongst themselves, rather than being equally reflective of an underlying latent construct - has been empirically tested by the development and application of data analytic techniques known as 'network analysis' (Borsboom \& Cramer, 2014; Boschloo et al., 2015; Frewen, Schmittmann, Bringmann \& Borsboom, 2013; Fried et al., 2015). From this perspective, symptoms are not reflective of an underlying disorder; instead, the associations among 
symptoms constitute the disorder. Networks are comprised of nodes (e.g., symptoms) and edges (associations among symptoms). Importantly, a network perspective does not assume symptoms to be interchangeable, but instead allows for the examination of the importance or centrality of symptoms empirically. Highly connected items that are likely to spread activation through the symptom network once activated are more central, whereas items with fewer connections lie on the periphery of a network and are less important (Cramer et al., 2012; Fried et al., 2015; Borsboom \& Cramer, 2013).

To date, only a single study has employed a network analysis of PTSD symptoms (McNally et al., 2015). In this study of 362 survivors of the Wenchuan earthquake in China, key findings included: the centrality of the hypervigilance symptom; connections between anger, sleep, irritability, and concentration difficulties; and associations among intrusive thoughts, dreams, and flashbacks. The study analyzed the DSM-IV PTSD symptoms. In the current study, we estimated networks of PTSD symptoms using data from the National Health and Resilience in Veterans Study (NHRVS), which is a contemporary, nationally representative study of U.S military veterans. We extended the McNally et al. (2015) study in four ways: (1) for the first time, we constructed PTSD networks based on the DSM-5 rather than the DSM-IV criterion symptoms; (2) we employed a sub-sample of veterans reporting clinically significant PTSD symptoms from a representative U.S. veteran study; pertinent given the plethora of research detailing generally higher rates of symptomatology and comorbidity in this population (Giacco et al., 2013; Jakupcak et al., 2011; Karstoft et al., 2014; Tsai et al., 2014); (3) we estimated the network structure among PTSD symptoms, and also, for the first time, added a number of clinical covariates (SI, depression, anxiety, physical QoL, mental QoL, age, and gender) into the network to examine whether they are differentially related to PTSD symptoms; and (4) we analyzed the robustness and stability of the networks. 


\section{Methods}

\subsection{Sample}

\subsubsection{Participants}

A total of 1,484 (mean age $=60.4$ years, $S D=15.3$, range $=20-94)$ veterans aged 21 years and older participated in the second baseline cohort of the National Health and Resilience in Veterans Study (NHRVS), conducted from September-October 2013. The sample was collected using KnowledgePanel, a nationally representative survey research panel of more than 50,000 adults that represents approximately $98 \%$ of all U.S. households. KnowledgePanel is maintained by GfK, a survey research company based in Menlo Park, California, that uses probability-based sampling of addresses from the U.S. Postal Service's Delivery Sequence File (DSF) in its participant recruitment, including households both with and without telephone numbers and/or Internet access. Veterans completed a 60-minute web-based survey that assessed a range of sociodemographic, psychiatric, and health variables. A total of 221 veterans met inclusion criteria (described below in detail) and were analyzed in this study.

\subsubsection{Measures}

Trauma Exposure and PTSD Symptoms. Veterans were administered the Trauma History Screen (THS; Carlson, Smith \& Palmieri, 2011), which asked whether they had been exposed to any of 14 potentially traumatic events in their lifetimes. Veterans who endorsed multiple trauma exposures were asked, "Which of these experiences was the worst for you?". The Posttraumatic Stress Disorder Checklist for DSM-5 (PCL-5; Weathers et al., 2013) was used to assess past-month symptoms of PTSD in relation to this worst event. The PCL-5 consists of 20 items (total score range: $0-80$ ) that inquire about the extent to which an individual is bothered by each of the DSM- 
5 PTSD symptoms on a 5-point Likert scale: $0=$ Not at all; $1=$ A little bit; $2=$ Moderately; $3=$ Quite a bit; 4=Extremely. Cronbach's alpha on PCL-5 items in the current sample was excellent (0.90).

Depression and anxiety symptoms. Current depression and anxiety symptoms were assessed using the Patient Health Questionnaire-4 (PHQ-4; Kroenke, Spitzer, Williams, \& Löwe, 2009), a 4-item self-report screening instrument for depression and anxiety. Each construct is measured by two items. These items are summed and scores $\geq 3$ on the depression and anxiety items are indicative of positive screens for depression and anxiety.

Suicidal ideation. SI in the past two weeks was assessed using two questions, adapted from the SI question in the Patient Health Questionnaire-9 (PHQ-9; Kroenke \& Spitzer, 2002), to assess both passive and active SI (Thompson, Henkel, \& Coyne, 2004). Specifically, respondents were asked: "How often have you been bothered by thoughts you might be better off dead?" (passive SI) and "How often have you been bothered by thoughts of hurting yourself in some way?" (active SI). Since the current study already has a comparably large number of nodes compared to the number of participants, and because the correlation between active and passive SI was high (0.73), we only included active SI, which was coded as a response $\geq 1$ (Several days).

Mental and Physical Functioning / QoL. The Short Form-8 Health Survey (SF-8; QualityMetric, 2014) measures QoL, functional health and well-being. It is a validated, abbreviated version of the SF-12 Health Survey (Ware,Kosinski, Turner-Bowker, \& Gandek, 2002) and one of the most widely used measures of physical and mental functioning / QoL. Component mental and physical health summary scores range from $0-100$, with a score of 50 representing the average level of 
functioning in the general population with each 10-point interval representing one standard deviation. Higher scores reflect better functioning.

\subsection{Missing Data and Sample Derivation}

PCL-5 responses were used to identify veterans with clinically significant PTSD symptoms according to the following criteria: 1) probable past-month DSM-5 PTSD, defined by a total score of $>38$ on the PCL-5 (Hoge, Riviere, Wilk, Herrell, \& Weathers, 2014); or 2) probable subthreshold PTSD, defined as endorsement of 2 or 3 B-E criteria, or endorsement of all 4 criteria but with total PCL-5 scores of under 38 (McLaughlin et al., 2015). Item-level data were missing for $1.6 \%$ of the sample and were imputed using an iterative Markov chain Monte Carlo (MCMC) method. A total of 221 veterans met either of the above criteria: 59 (weighted 27.7\%) for probable past-month DSM-5 PTSD and 162 (weighted 72.3\%) for probable subthreshold DSM-5 PTSD. Data from these 221 veterans were analyzed in this study.

\subsection{Data Analysis}

Data and analytic code for this manuscript are available in the Online Supplementary Materials.

2.3.1 Network estimation. We estimated the structure of three networks, using the $R$ package qgraph. In a first step, we included the 20 PTSD symptoms in the estimation procedure (N1). Second, we added seven clinical covariates to the network that are outlined in detail above (N2): SI, depression, anxiety, physical QoL, mental QoL, age, and gender. In general, networks feature nodes (in our case: symptoms and clinical covariates) and edges (the connections among nodes). A main advantage of the network approach is that it visualizes the multivariate dependencies of the data that otherwise remain hidden. The most informative visualization, which 
we also choose here, is one in which edges can be understood as partial correlation coefficients, meaning that a connection between nodes A and B is the connection after controlling for all other edges in the network. To do so, we estimated a Gaussian Graphical Model that estimates pairwise association parameters between all nodes. With 20 symptom nodes, 190 pairwise association parameters are estimated, and 351 pairwise association parameters for the second network with 27 nodes. The estimation of that many parameters likely leads to a number of false positive connections, and we control for these by using the least absolute shrinkage and selection operator (LASSO) (Tibshirani, 1996) that sets very small edges to zero. In other words, the LASSO procedure employs a regularization technique that conservatively identifies only the relevant edges, and accurately discovers the underlying network structure (van Borkulo et al., 2014). More details on the estimation of such regularized partial correlation networks, including a tutorial that explains how to estimate such models in R, is available elsewhere (Epskamp \& Fried, 2016). Since PTSD symptoms can be considered ordered-categorical, the estimation of the 20 -symptom network is based on the polychoric correlation among symptoms. For the 27 -item network that includes covariates, we used the cor_auto function of the qgraph package that automatically computes the appropriate correlations for different variable types (polychoric, polyserial, or Pearson).

In a last step, we examined the impact of covariates on the associations among the 20 PTSD symptoms. To do so, we deleted the 7 covariates from the adjacency matrix of the full 27 -node network, which results in the connections among the 20 PTSD symptoms controlling for the 7 covariates $(\mathrm{N} 2 *)$. We subtracted this modified adjacency matrix $\mathrm{N} 2 *$ from the adjacency matrix of N1 (the 20 PTSD symptoms, not controlling for covariates) to derive a delta network (N3) that contains the change of N1 upon including the covariates.

2.3.2 Centrality estimation. For the 20-item PTSD symptom network, we were interested which symptoms are most relevant. There are three common graph theoretical centrality measures 
(Opsahl, Agneessens, \& Skvoretz, 2010). (1) Node strength sums all edges of a given symptom with all other symptoms, estimating how strongly a node is directly connected with the network. (2) Closeness centrality provides a measure of how strongly a node is connected indirectly with the network by taking the inverse of all shortest path lengths between a node and all other nodes. (3) Betweenness centrality relies on the concept of shortest path length connecting any two symptoms, and a symptom with a high betweenness centrality can be considered to be central in connecting other symptoms.

2.3.3 Robustness estimation. When estimating psychopathological networks, a major current challenge is that the stability and accuracy of psychopathological networks - and derived graph theoretical measures such as centrality—is unclear (Epskamp, Borsboom, \& Fried, 2016). To meet this challenge, we performed two robustness analyses using the novel R-package bootnet. First, we bootstrapped the confidence regions of the edge weights, providing an estimate about the certainty and precision of edges in the networks. Second, we examined the stability of the order of the centrality estimation by subsetting bootstrap. Edge weight bootstrap was applied to both networks, subsetting bootstrap only to the 20 item PTSD network (since this is the network we are interested in centrality). A more detailed explanation of these techniques, along with their results, can be found in the supplementary materials. An R-tutorial paper on stability of psychopathological networks, and the R-package bootnet, is available elsewhere (Epskamp et al., 2016).

2.3.4 Visualization. Positive edges are printed in green, negative ones in red, and the stronger a connection, the thicker and more saturated it is. We use the Fruchterman-Reingold algorithm (Fruchterman \& Reingold, 1991) that places nodes with stronger and/or more connections more closely together. We set the maximum edge value across all networks to 0.45 , the strongest edge identified across networks. This means saturation and thickness of edges can be 
compared across networks (equally thick edges across networks have equal edge weights). We used a minimum value of 0.03 in all networks to enhance the interpretability of the graphs.

\section{Results}

\subsection{Sample Characteristics}

Veterans ranged in age at the time of assessment from 21-89 years, the mean age was 54.0 years $(S D=14.8)$, and the majority $(86.7 \%)$ were male and combat veterans $(n=107$; weighted 54.0\%). PCL-5 scores reflecting DSM-5 PTSD symptoms ranged from 4-80 $(M=31.0 ; S D=13.4)$. A total of 61 veterans (weighted 26.2\%) endorsed SI. Trauma exposures ranged between 1 and 15 events, with the average number of exposures being $6.0(S D=3.2)$; the traumatic events were on average 21.5 years ago $(S D=18.2$ years, range $0-70$ years). The three most commonly endorsed 'worst' traumatic events were: sudden death of close family member or friend $(n=49$; weighted $24.8 \%$ ); saw something horrible or was badly scared during military service $(n=28$; weighted $16.0 \%)$; and suddenly abandoned by spouse, partner, parent, or family ( $n=24$; weighted $11.9 \%)$.

\subsection{PTSD network}

Figure 1 shows a visualization of the network structure of the 20 DSM-5 PTSD symptoms. Overall, symptoms were positively connected within the network, and especially strong connections emerged between nightmares (B2) and flashbacks (B3), blame of self or others (D3) and negative trauma-related emotions (D4), detachment (D6) and restricted affect (D7), and hypervigilance (E3) and exaggerated startle response (E4). The edge weight bootstrap procedure revealed confidence intervals of moderate size around the edges weights (see Figure S1 in the supplementary materials). 


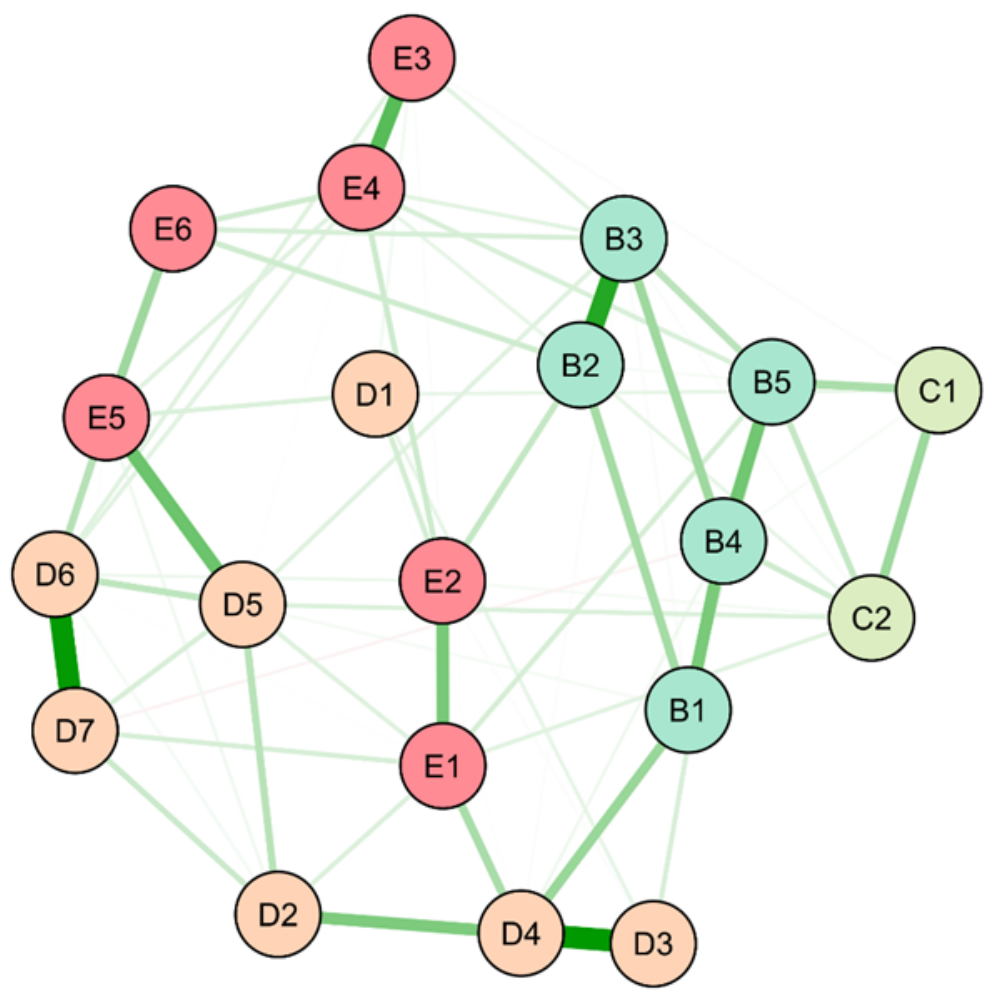

B1 - Intrusive thoughts

B2 - Nightmares

B3 - Flashbacks

B4 - Emotional cue reactivity

B5 - Physiological cue reactivity

C1 - Avoidance of thoughts

C2 - Avoidance of reminders

D1 - Trauma-related amnesia

D2 - Negative beliefs

D3 - Blame of self other others

D4 - Negative trauma-related emotions

D5 - Loss of interest

D6 - Detachment

D7 - Restricted affect

E1 - Irritability/anger

E2 - Self-destructive/reckless behavior

E3 - Hypervigilance

E4 - Exaggerated startle response

E5 - Difficulty concentrating

E6 - Sleep disturbance

Figure 1: Network containing the 20 DSM-5 symptoms of PTSD. Green lines represent positive associations, red lines negative ones, and the thickness and brightness of an edge indicate the association strength.

The standardized estimates of betweenness, closeness, and node strength centrality are presented in Figure 2; centrality estimates were substantially interrelated (correlation of 0.65 between strength and closeness, 0.72 between strength and betweenness, and 0.82 between closeness and betweenness). In the robustness analyses, only the order of node strength centrality was estimated with a moderate amount of precision, while the order of betweenness and closeness centrality should be interpreted with more care (Figures S2 and S3 in the supplementary materials). We therefore focus our interpretation of the most relevant symptoms on node strength centrality in the remainder of the report, consistent with other work showing that node strength is the most robustly estimated metric in network analyses. 
The five nodes with the highest node strength centrality were negative trauma-related emotions (D4), detachment (D6), physiological cue reactivity (B5), flashbacks (B3), and emotional cue reactivity (B4), whereas the two least central nodes (with a substantial drop in node strength) were avoidance of thoughts (C1) and trauma-related amnesia (D1). This does not come as a surprise, considering that these two symptoms only exhibit few and weak connections (see Figure 1).

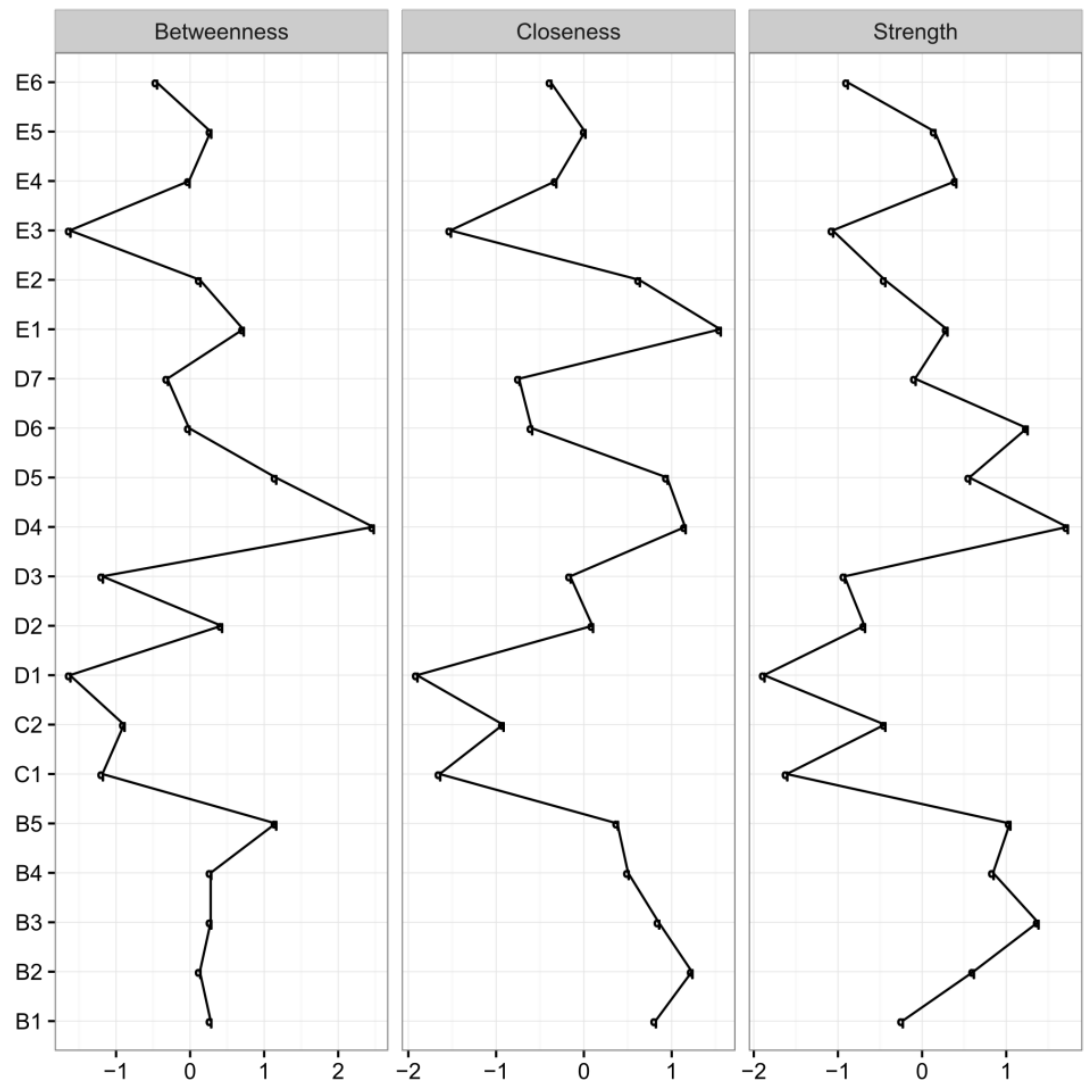

Figure 2: Betweenness, closeness, and node strength centrality estimates for the 20 DSM-5 PTSD criterion symptoms. See Figure 1 for symptom descriptions of the shortcodes.

\subsection{PTSD network with clinical covariates}


In a second step, we estimated the network structure of the 20 DSM-5 PTSD symptoms, with seven clinical covariates incorporated into the model. The resulting network is visualized in Figure 3; similar to the 20-item DSM-5 PTSD network, moderate confidence intervals around the edge weights were found in the robustness analyses (see Figure S4 in the supplementary materials).

It is important to note once again that this graph can be understood as a partial correlation network: the associations are the ones left over when controlling for all other associations in the network. In this sense, edges between covariates and symptoms, for instance between suicidal ideation (SI) and self-destructive behavior (E2), are meaningful because they are not explained by the covariation among PTSD symptoms alone. Relevant associations emerged between covariates and symptoms (which can be interpreted as partial correlations) between self-destructive behavior (E2) and SI (0.25), between difficulty concentrating (E5) with anxiety (Anx; 0.13), depression (Dep; 0.12) and mental QoL / functioning (MFunc; -0.11), and between depression (Dep) with restricted affect (D7; 0.13). Of note, some of the covariates were substantially interrelated, including age with sex (-0.26), mental functioning (MFunc) with anxiety (Anx: -.28) and depression (Dep: -.24), depression (Dep) with suicidal ideation (SI: 0.39), and anxiety (Anx) with depression (Dep: 0.25). 

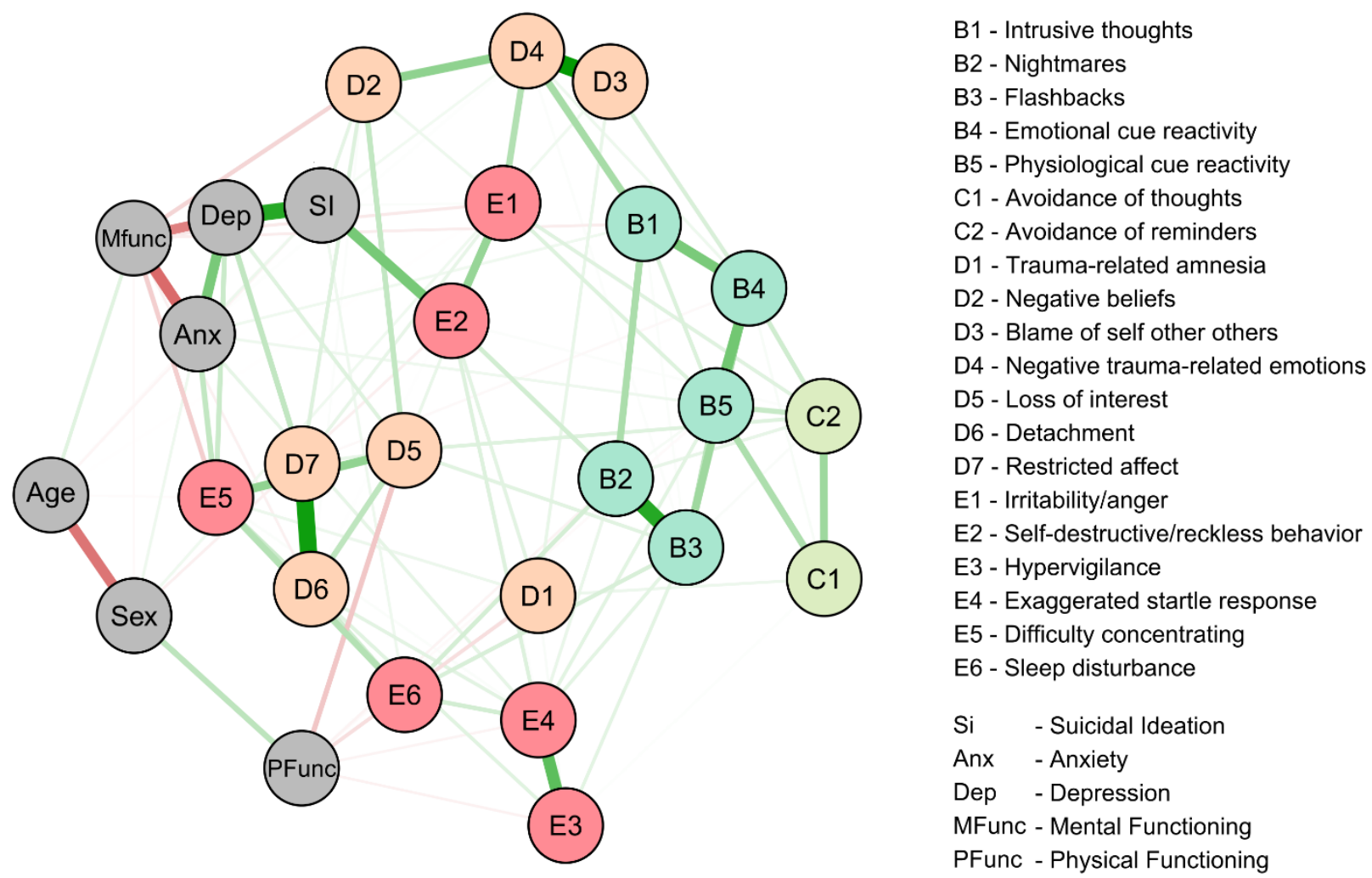

Figure 3: Network containing the 20 DSM-5 symptoms of PTSD, along with 7 clinical covariates. Green lines represent positive associations, red lines negative ones, and the thickness and brightness of an edge indicate the association strength.

\subsection{Impact of covariates on the relations among symptoms}

Finally, we examined the impact of including the 7 covariates on the connections among PTSD symptoms. The resulting delta network in Figure 4 is nearly empty and features few very weak edges; the strongest edge weight was only 0.06 . This implies that connectivity among symptoms changes very little upon including the clinical covariates in the system. The sum of edges of the 20 symptoms was reduced from 8.7 to 7.7 once controlling for covariates; covariates "explain away" only about $11.5 \%$ of the connectivity of the PTSD symptoms. This conclusion is further corroborated by a very high correlation of 0.97 between the edge weights across the two networks 
subtracted to obtain the delta network (see Methods section). In sum, the 20-item DSM-5 PTSD network is robust when other relevant variables are entered into the network and remains largely unaffected.

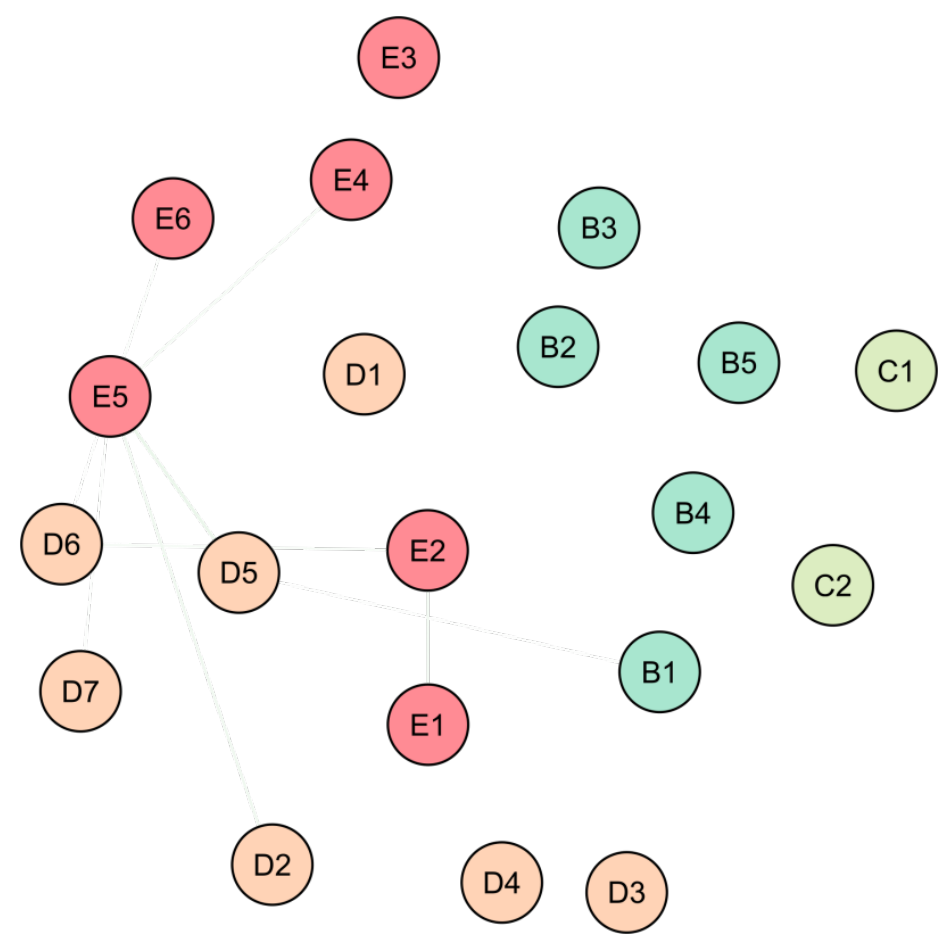

Figure 4: Change of the relations among 20 DSM-5 criterion symptoms of PTSD in Figure 1 upon entering seven relevant clinical covariates (not presented). Green lines represent positive associations, red lines negative ones, and the thickness and brightness of an edge indicate the association strength.

\section{Discussion}

To the best of our knowledge, the current study represents the first network analysis of DSM-5 PTSD symptoms (APA, 2013). We (1) analyzed 221 individuals with clinically significant PTSD symptoms drawn from a representative sample of U.S. military veterans, (2) estimated the 
structure of two networks (a 20-item DSM-5 PTSD symptom network and a 27-item network adding seven clinical covariates), and (3) tested the robustness and stability of the networks. We believe these three aspects add to the small body of literature on PTSD networks (McNally et al., 2015) and provide pertinent insights into the complex relationships between PTSD symptoms and clinically relevant correlates. Below we will first discuss connectivity between symptoms and symptom centrality in the PTSD network. Second, we will discuss the PTSD network with clinical covariates.

\subsection{PTSD network}

In the current study, DSM-5 PTSD symptoms were generally positively connected among each other. Especially strong associations emerged between nightmares (B2) with flashbacks (B3); blame of self or others (D3) with negative trauma-related emotions (D4); detachment (D6) with restricted affect (D7); and hypervigilance (E3) with exaggerated startle response (E4). Of note, each of these connected symptom pairs as noted above co-occur within the network and therefore may appropriately belong to a particular symptom cluster within the DSM-5. However, some symptoms provided weak, and at times absent, connections with others from their respective DSM5 symptom groups. For example, few connections were found between psychogenic amnesia (D1) and other symptoms from the NACM cluster (group D in Figure 1). From a network perspective, the absence of a connection between two symptoms implies that they are conditionally independent of each other given the other symptoms in the network. If this finding were to generalize in other samples, it would raise doubts whether psychogenic amnesia (D1) is indeed a good fit for the NACM symptom group. Our result is consistent with prior reports: repeatedly, weak factor loadings of psychogenic amnesia within the PTSD confirmatory factor analytic literature have emerged, which have led researchers to question whether this item should be considered a core PTSD symptom (cf. Armour et al., 2016). 
There were a number of similarities between our results and those of the only prior PTSD network analysis study (McNally et al., 2015). In both studies, strong associations were observed between the hypervigilance (E3) and exaggerated startle response (E4) symptoms. Of note, McNally and colleagues also suggested that this association may be a self-reinforcing feedback loop. In addition, nightmares (B2) and flashbacks (B3) were strongly associated. These connections align with the clustering of PTSD symptoms as set out in both the DSM-5, given that hypervigilance (E3) and exaggerated startle response (E4) cluster in the alterations in arousal and reactivity symptom group (AAR), and nightmares (B2) and flashbacks (B3) are within the intrusions symptom cluster. Unlike McNally et al. (2015), we discovered additional associations with the two remaining re-experiencing symptoms of emotional (B4) and physiological cue reactivity (B5). Indeed, in our network, although the strength of connections varied, most reexperiencing symptoms were linked either directly or indirectly (through other symptoms [nightmare were linked to emotional cue reactivity (B4) through flashbacks (B3)]) with each other. This is also a notable finding given that the symptoms comprising the re-experiencing symptom group remained unchanged from the DSM-IV to DSM-5.

Blame of self or others (D3) and negative trauma-related emotions (D4) were highly connected in our network, and negative trauma-related emotions (D4) was connected, albeit to a lesser degree, with negative beliefs (D2). The connections between these symptoms are intuitive and may have relevance in the context of treatment planning. For example, cognitive processing therapy (CPT) is regarded as a highly effective way of treating chronic PTSD in veterans (Monsoon et al., 2006). CPT specifically targets thoughts, feelings, and beliefs about the PTSD precipitating traumatic event. Thus, if these symptoms are shown to be central to a PTSD network, the targeting of these symptoms within treatment planning could be particularly effective and this should be investigated in future research. 
Assessing the robustness of symptom centrality revealed that the most central symptoms did not differ substantially from each other in their centrality, and should be considered roughly equally important. The most central symptoms were negative trauma-related emotions (D4), flashbacks (B3), detachment (D6), and physiological cue reactivity (B5), and we conclude that they may be of greatest clinical significance in U.S. military veterans. These symptoms differ considerably from the most central symptoms identified by McNally et al. (2015), who identified hypervigilance, concentration difficulties, and dreams about the trauma and future foreshortening as most central in their network model of DSM-IV PTSD symptoms. The fact that studies differ in the trauma populations under investigation (e.g., McNally et al. focused on PTSD symptoms related to a specific event [earthquake], while our sample focused on PTSD symptoms related to a broad range of traumatic events with the most common type of event reported as bereavement and the third most common as abandonment) could explain the differences between studies, along with the use of DSM-IV vs. DSM-5 criteria. Since PTSD symptom presentation can differ based on the precipitating traumatic event (Armour \& Shevlin, 2010), it stands to reason that PTSD symptom networks and their associated findings may differ based on the index traumas of the sample under investigation. This has previously been shown for networks among depression symptoms, which can differ depending on precipitating life events such as romantic loss, stress, or losing a loved one (Cramer, Borsboom, Aggen, \& Kendler, 2013; Fried et al., 2015).

Identifying the symptom/s that are most central to a PTSD network is, however, an important endeavor given that a reduction in a central symptom could in turn alleviate a wide range of symptoms ultimately reducing the overall psychological burden of PTSD symptomology. Indeed, follow up research should investigate whether targeting these specific symptoms in interventions may decrease the burden and suffering of PTSD symptoms U.S. military veterans, as well as other trauma-affected populations. 


\subsection{PTSD network with clinical covariates}

In extending our 20-item DSM-5 PTSD network, we added seven additional clinically relevant variables to the network. The strong association between self-destructive behavior (E2) and suicidal ideation (SI) is unsurprising given previous reports of self-destructive behaviors predicting males' SI (Hopes \& Williams, 1999); prior research revealing that both thoughts of ending one's life and a previous suicide attempt significantly correlated with a diagnosis of PTSD in Vietnam veterans $(r=.53, \mathrm{p}<.001$; and $\mathrm{r}=.33, \mathrm{p}<.001$, respectively; Kramer et al., 1994); and that out of six anxiety based psychiatric disorders, PTSD alone was significantly associated with suicidal ideation or attempts (Sareen, Houlaghan, Cox, \& Asmundson, 2005). Of note, the selfdestructive behavior item is a new addition to PTSD's nosology in the DSM-5, and our results suggest that this symptom may be a key contributor to SI risk in veterans. Some of the covariates in the 27-item network were substantially inter-related, the strongest of which was between depression and SI. While the causal direction of this association remains unclear, this link speaks to the possibility of a potential increased risk for suicidal ideation in trauma survivors displaying comorbid symptoms of depression and heightened self-destructive behaviors. The relationship between depression and suicide has been previously reported with individuals experiencing depression being at a much greater risk of suicide than members of the general population (Kessler et al., 1994) and reports that approximately $2-7 \%$ of patients who are treated for depression die by suicide (Bostwick \& Pankratx, 2000; Inskip, Harris, \& Barraclough). Another strong association among clinical covariates was identified among depression and anxiety symptoms. This is consistent with several prior network studies reporting inter-relatedness of the depression and anxiety constructs (Boschloo et al., 2015; Cramer et al., 2010). A further notable finding was that concentration difficulties (E5) were connected with anxiety (Anx) and depression (Dep) symptoms, and mental QoL / functioning (Mfunc). Prior research has suggested that items such as difficulty 
concentrating are not core symptoms of PTSD but rather reflect a more general distress component (Elklit, Armour, \& Shevlin, 2010; Simms, Watson, \& Doebbeling, 2002). The fact that concentration difficulties connect with multiple related constructs would support this viewpoint. Similarly, depression was found to be connected with restricted affect (D7).

\subsection{Conclusions and future directions}

This study generates two intriguing questions; 1. Is the co-occurrence among PTSD symptoms better explained by a model of mutually reinforcing symptoms instead of the more commonly used factor models based on the common cause hypothesis? and 2. Are these models really mutually exclusive or could a trauma serve as the common cause for some symptoms initially that then mutually interact over time in a causal system? Thus, the current study provides an extremely useful tool to generate hypotheses for follow-up studies.

Overall, the study provides further clarity in regard to how the DSM-5 PTSD symptoms relate to each other. Adding covariates to the network results in very little change in associations among PTSD symptoms, providing evidence that these connections can be considered fairly robust. A major field of future research are the DSM-5 PTSD symptom groups. While the network approach merely understands such clusters as groups of elements that are somewhat more associated amongst each other, they play a crucial role in the DSM-5 given that diagnostic algorithms are directly linked to symptom groups. They determine who does and does not receive a diagnoses and in turn access to services; future analyses of the covariance among the clusters are hence of utmost importance to inform potential revisions of the DSM. One line of enquiry could be the investigation of a particular PTSD symptom factor by dropping the one variable/symptom that has shown very low centrality in our study; with a view to determining if doing so would improve fit. 
The identification of a group of highly connected symptoms (D2-D4) has a number of additional clinical implications. If it is known that certain symptoms are highly connected and central within a network, then these symptoms can be targeted in treatment planning. The alleviation of a highly connected symptom/s may ultimately break down the overall PTSD network resulting in significant treatment gains. Future research should also endeavor to identify which symptoms drive the course of PTSD longitudinally. If these symptoms are targeted and alleviated, then this may halt the sometimes chronic nature of PTSD. In a similar vein, if certain symptoms within a PTSD network are identified as acting as key symptoms (known as bridge symptoms) leading to comorbid psychological disorders or behavioral outcomes of distress, then these symptoms can be targeted and alleviated to prevent comorbidity and/or adverse outcomes such as suicidal ideation, behavior, and attempts. In the current study, self-destructive behavior was identified as being associated with suicidal ideation, future studies of this nature should further explore this relationship, preferably by conducting finer grained analyses of the association between PTSD symptoms and multiple indicators of suicidal ideation and behavior. Additionally, these connections, both in a PTSD network and in one which also incorporates other related constructs and behaviors, should be assessed across a wide range of trauma populations to determine commonalities and differences depending on the index trauma. It would be of clinical relevance to know the manner of these connections in the index trauma of your patients as such would greatly facilitate treatment planning.

\subsection{Limitations}

The results have to be interpreted in the light of a number of limitations. First, the robustness analyses revealed that there was moderate uncertainty regarding the estimation of the edge weights and centrality parameters. This means that, for instance, while the few DSM-5 PTSD symptoms with the highest centrality estimates were very likely more central than the few 
symptoms at the lower end, we cannot properly distinguish which symptoms were more central among the top or among the bottom symptoms. The same holds for edge weights: while the confidence intervals of the strongest positive and negative edges do not overlap with the majority of moderately strong, weak, or absent edges, distinguishing clearly among the many moderately strong edges is not possible. Second, given that we utilized cross-sectional data it is impossible to ascertain directed influences among symptoms. Further, we also cannot distinguish between indegree (symptoms are triggered by other symptoms) and outdegree centrality (symptom activate other symptoms), which requires longitudinal data (e.g., Bringmann, Lemmens, Huibers, Borsboom, \& Tuerlinckx, 2015). Third, our data was collected via a web-based, self-report assessment; future studies of this nature may wish to assess PTSD symptom networks from data collected via structured clinical interviews (Clinician-Administered PTSD Scale for DSM-5). Fourth, while the network approach follows the Research Domain Criteria (RDoC) set by the National Institute of Mental Health (NIMH) in assessing specific symptoms that can be considered distinct endophenotypes, it would be interesting to capture more RDoC-relevant dimensions such as physiology, behavior and self-reports in future studies. Fifth, there are possibly many individual symptom networks dependent on the person and their particular trauma, and the present crosssectional group-level analysis cannot uncover this type of heterogeneity. We believe that network analysis in larger groups of traumatized individuals may provide a great starting point to understand how PTSD symptoms relate to each other on average, but that future studies should focus on the idiographic aspects of trauma which may lead to a personalized medicine approach to understanding PTSD. Finally, the current study assessed the PTSD network and associations pertaining to individuals who displayed clinically significant symptomatology, rather than, for example, the whole trauma exposed sample which would have included those with little to no PTSD symptomatology. This more restrictive sampling led to comparably little statistical power 
when estimating the networks. For this reason, we applied the novel bootstrap routine to test the stability of the networks, and assessed covariates such as depression and anxiety as single nodes rather than examining covariance among individual symptoms of these constructs with the PTSD symptoms. Future research may endeavor to apply network analysis in larger clinical samples and examine comorbidity at the symptom level across all psychopathological constructs. 


\section{References}

Armour, C., Müllerová, J., \& Elhai, J. D. (2016). A systematic literature review of PTSD's latent structure in the diagnostic and statistical manual of mental disorders: DSM-IV to DSM-5. Clinical Psychology Review, 44, 60-74.

Alman, L. G., Guffanti, G., Fan, B., Duarte, C., Wu, P., Musa, G., .. \& Hoven, C. (2012). O-15Latent class analysis of PTSD symptoms among 6,733 New York City students exposed to 9/11. European Psychiatry, 27, 1.

American Psychiatric Association. (2013). Diagnostic and statistical manual of mental disorders (5th ed.). Arlington, VA: Author.

Asmundson, G. J., Stapleton, J. A., \& Taylor, S. (2004). Are avoidance and numbing distinct PTSD symptom clusters?. Journal of Traumatic Stress, 17(6), 467-475.

Au, T. M., Dickstein, B. D., Comer, J. S., Salters-Pedneault, K., \& Litz, B. T. (2013). Cooccurring posttraumatic stress and depression symptoms after sexual assault: a latent profile analysis. Journal of Affective Disorders, 149(1), 209-216.

Ayer, L., Danielson, C. K., Amstadter, A. B., Ruggiero, K., Saunders, B., \& Kilpatrick, D. (2011). Latent classes of adolescent posttraumatic stress disorder predict functioning and disorder after 1 year. Journal of the American Academy of Child \& Adolescent Psychiatry, 50(4), 364-375.

Bonanno, G. A., Papa, A., Lalande, K., Westphal, M., \& Coifman, K. (2004). The importance of being flexible the ability to both enhance and suppress emotional expression predicts long-term adjustment. Psychological Science, 15(7), 482-487.

Borsboom, D., \& Cramer, A. O. (2013). Network analysis: an integrative approach to the structure of psychopathology. Annual Review of Clinical Psychology, 9, 91-121.

Borsboom, D. (2008). Psychometric perspectives on diagnostic systems. Journal of Clinical Psychology, 64(9), 1089-1108.

Boschloo, L., van Borkulo, C. D., Rhemtulla, M., Keyes, K. M., Borsboom, D., \& Schoevers, R. A. (2015). The Network Structure of Symptoms of the Diagnostic and Statistical Manual of Mental Disorders. PloS one, 10(9), e0137621.

Bostwick, J. M., \& Pankratz, V. S. (2000). Affective disorders and suicide risk: a re-examination. American Journal of Psychiatry, 157,1925-1932.

Brady, K. T., Killeen, T. K., Brewerton, T., \& Lucerini, S. (2000). Comorbidity of psychiatric disorders and posttraumatic stress disorder. The Journal of Clinical Psychiatry, (supp1 7), 1-478.

Breslau, N., Reboussin, B. A., Anthony, J. C., \& Storr, C. L. (2005). The structure of posttraumatic stress disorder: latent class analysis in 2 community samples. Archives of General Psychiatry, 62(12), 1343-1351. 
Bringmann, L. F., Lemmens, L. H., Huibers, M. J., Borsboom, D., \& Tuerlinckx, F. (2015). Revealing the dynamic network structure of the Beck Depression Inventory-II. Psychological Medicine, 45(04), 747-757.

Carlson, E. B., Smith, S. R., Palmieri, P. A., Dalenberg, C., Ruzek, J. I., Kimerling, R., ... \& Spain, D. A. (2011). Development and validation of a brief self-report measure of trauma exposure: the Trauma History Screen. Psychological Assessment, 23(2), 463.

Contractor, A. A., Durham, T. A., Brennan, J. A., Armour, C., Wutrick, H. R., Frueh, B. C., \& Elhai, J. D. (2014). DSM-5 PTSD's symptom dimensions and relations with major depression's symptom dimensions in a primary care sample. Psychiatrc Research, 215(1), 146-153.

Cramer, A. O., Waldorp, L. J., van der Maas, H. L., \& Borsboom, D. (2010). Comorbidity: A network perspective. Behavioral and Brain Sciences, 33(2-3), 137-150.

Cramer, A. O. J., Borsboom, D., Aggen, S. H., \& Kendler, K. S. (2013). The pathoplasticity of dysphoric episodes: differential impact of stressful life events on the pattern of depressive symptom inter-correlations. Psychological Medicine, 42(5), 957-65.

doi:10.1017/S003329171100211X

Creamer, M., Burgess, P., \& McFarlane, A. C. (2001). Post-traumatic stress disorder: findings from the Australian National Survey of Mental Health and Well-being. Psychological Medicine, 31(07), 1237-1247.

Dickstein, B. D., Suvak, M., Litz, B. T., \& Adler, A. B. (2010). Heterogeneity in the course of posttraumatic stress disorder: trajectories of symptomatology. Journal of Traumatic Stress, 23(3), 331-339.

DSM-5 American Psychiatric Association. (2013). Diagnostic and statistical manual of mental disorders. Arlington: American Psychiatric Publishing.

Elklit, A., Armour, C., \& Shevlin, M. (2010). Testing alternative factor models of PTSD and the robustness of the dysphoria factor. Journal of Anxiety Disorders, 24(1), 147-154.

Epskamp, S., Borsboom, D., \& Fried, E.I. (2016). Estimating Psychological Networks and their Stability: a Tutorial Paper. Arxiv Preprint (ID 160408045) 1-34.

Epskamp, S., \& Fried, E.I. (2016). A Primer on Estimating Regularized Psychological Networks. Arxiv Preprint (ID 160701367) 1-21.

Epskamp, S., Maris, G., Waldorp, L. J., \& Borsboom, D. (2015). Network psychometrics. Handbook of Psychometrics. New York: Wiley. 
Frewen, P. A., Schmittmann, V. D., Bringmann, L. F., \& Borsboom, D. (2013). Perceived causal relations between anxiety, posttraumatic stress and depression: extension to moderation, mediation, and network analysis. European Journal of Psychotraumatology, 4.

Fried, E.I., 2015. Problematic assumptions have slowed down depression research: why symptoms, not syndromes are the way forward. Front Psychol 6, 1-11.

Fried, E. I., Bockting, C., Arjadi, R., Borsboom, D., Tuerlinckx, F., Cramer, A., Epskamp, S., Amshoff, M., Carr, D., \& Stroebe, M. (2015). From Loss to Loneliness: The Relationship Between Bereavement and Depressive Symptoms. Journal of Abnormal Psychology, 124, 256265.

Fruchterman, T. M., \& Reingold, E. M. (1991). Graph drawing by force-directed placement. Software: Practice and Experience, 21(11), 1129-1164.

Galatzer-Levy, I. R., \& Bryant, R. A. (2013). 636,120 ways to have posttraumatic stress disorder. Perspectives on Psychological Science, 8(6), 651-662.

Giacco, D., Matanov, A., \& Priebe, S. (2013). Symptoms and subjective quality of life in posttraumatic stress disorder: a longitudinal study. PloS one, 8(4), e60991.

Hoge, C. W., Riviere, L. A., Wilk, J. E., Herrell, R. K., \& Weathers, F. W. (2014). The prevalence of post-traumatic stress disorder (PTSD) in US combat soldiers: a head-to-head comparison of DSM-5 versus DSM-IV-TR symptom criteria with the PTSD checklist. The Lancet Psychiatry, 1(4), 269-277.

Hopes, L. M., \& Williams, A. (1999). Depression, self-defeating, and self-destructive behaviors as predictors of suicide ideation in males and females. Psychological Reports, 84(1), 63-66.

Inskip, H. M., Harris, E. C., \& Barraclough, B. (1998). Lifetime risk of suicide for affective disorder, alcoholism and schizophrenia. The British Journal of Psychiatry, 172(1), 35-37.

Jakupcak, M., Hoerster, K. D., Varra, A., Vannoy, S., Felker, B., \& Hunt, S. (2011).

Hopelessness and suicidal ideation in Iraq and Afghanistan war veterans reporting subthreshold and threshold posttraumatic stress disorder. The Journal of Nervous and Mental disease, 199(4), 272-275.

Jakupcak, M., Cook, J., Imel, Z., Fontana, A., Rosenheck, R., \& McFall, M. (2009).

Posttraumatic stress disorder as a risk factor for suicidal ideation in Iraq and Afghanistan war veterans. Journal of Traumatic Stress, 22(4), 303-306.

Karam, E. G., Friedman, M. J., Hill, E. D., Kessler, R. C., McLaughlin, K. A., Petukhova, M., ... \& Girolamo, G. (2014). Cumulative traumas and risk thresholds: 12-month PTSD in the world mental health (WMH) surveys. Depression and Anxiety, 31(2), 130-142. 
Karstoft, K. I., Armour, C., Andersen, S. B., Bertelsen, M., \& Madsen, T. (2015). Community integration after deployment to Afghanistan: a longitudinal investigation of Danish soldiers. Social Psychiatry and Psychiatric Epidemiology, 50(4), 653-660.

Karstoft, K. I., Armour, C., Elklit, A., \& Solomon, Z. (2013). Long-term trajectories of posttraumatic stress disorder in veterans: the role of social resources. The Journal of Clinical Psychiatry, 74(12), 1-478.

Kessler, R. C., \& Üstün, T. B. (Eds.). (2008). The WHO World Mental Health Surveys: global perspectives on the epidemiology of mental disorders (pp. 1-580). New York: Cambridge University Press.

Kessler, R. C., McGonagle, K. A., Zhao, S., Nelson, C. B., Hughes, M., Eshleman, S., ... \& Kendler, K. S. (1994). Lifetime and 12-month prevalence of DSM-III-R psychiatric disorders in the United States: results from the National Comorbidity Survey. Archives of General Psychiatry, 51(1), 8-19.

Kessler, R. C., Sonnega, A., Bromet, E., Hughes, M., \& Nelson, C. B. (1995). Posttraumatic stress disorder in the National Comorbidity Survey. Archives of General Psychiatry, 52(12), 1048-1060.

Kessler, R. C., Rose, S., Koenen, K. C., Karam, E. G., Stang, P. E., Stein, D. J., ... \& McLean, S. A. (2014). How well can post-traumatic stress disorder be predicted from pre-trauma risk factors? An exploratory study in the WHO World Mental Health Surveys. World Psychiatry, 13(3), 265274.

Kramer, T. L., Lindy, J. D., Green, B. L., Grace, M. C., \& Leonard, A. C. (1994). The comorbidity of Post-traumatic stress disorder and suicidality in Vietnam veterans. Suicide and Life-Threatening Behavior, 24(1), 58-67.

Kroenke, K., Spitzer, R. L., \& Williams, J. B. (2002). The PHQ-15: validity of a new measure for evaluating the severity of somatic symptoms. Psychosomatic Medicine, 64(2), 258-266.

Kroenke, K., Spitzer, R. L., Williams, J. B., \& Löwe, B. (2009). An ultra-brief screening scale for anxiety and depression: the PHQ-4. Psychosomatics, 50(6), 613-621.

Lapierre, C. B., Schwegler, A. F., \& LaBauve, B. J. (2007). Posttraumatic stress and depression symptoms in soldiers returning from combat operations in Iraq and Afghanistan. Journal of Traumatic Stress, 20(6), 933-943.

Maguen, S., Madden, E., Bosch, J., Galatzer-Levy, I., Knight, S. J., Litz, B. T., ... \& McCaslin, S. E. (2013). Killing and latent classes of PTSD symptoms in Iraq and Afghanistan veterans. Journal of Affective Disorders, 145(3), 344-348.

McLaughlin, K. A., Koenen, K. C., Friedman, M. J., Ruscio, A. M., Karam, E. G., Shahly, V., \& Kessler, R. D. (2014). Subthreshold posttraumatic stress disorder in the World Mental Health (WMH) surveys. Biological Psychiatry. doi:10.1016/j.biopsych.2014.03.028. 
McNally, R. J., Robinaugh, D. J., Wu, G. W., Wang, L., Deserno, M. K., \& Borsboom, D. (2015). Mental disorders as causal systems a network approach to posttraumatic stress disorder. Clinical Psychological Science, 3(6), 836-849.

McNally, R. J. (2004). Conceptual problems with the DSM-IV criteria for posttraumatic stress disorder. In G. M. Rosen (Ed.), Posttraumatic stress disorder: Issues and controversies (pp. 114). New York: Wiley.

Monsoon, C. M., Schnurr, P.P., Resick, P.A., Friedman, M.T., Young-Xu, Y. \& Stevens, S.P. (2006). Cognitive processing therapy for veterans with military-related posttraumatic stress disorder. Journal of Consulting and Clinical Psychology, 74, 898-907.

Naifeh, J. A., Richardson, J. D., Del Ben, K. S., \& Elhai, J. D. (2010). Heterogeneity in the latent structure of PTSD symptoms among Canadian veterans. Psychological Assessment, 22(3), 666.

Opsahl, T., Agneessens, F., \& Skvoretz, J. (2010). Node centrality in weighted networks: Generalizing degree and shortest paths. Social Networks, 32(3), 245-251.

Pietrzak, R. H., Goldstein, R. B., Southwick, S. M., \& Grant, B. F. (2011). Prevalence and Axis I comorbidity of full and partial posttraumatic stress disorder in the United States: results from Wave 2 of the National Epidemiologic Survey on Alcohol and Related Conditions. Journal of Anxiety Disorders, 25(3), 456-465.

Pietrzak, R. H., Goldstein, R. B., Southwick, S. M., \& Grant, B. F. (2011). Prevalence and Axis I comorbidity of full and partial posttraumatic stress disorder in the United States: results from Wave 2 of the National Epidemiologic Survey on Alcohol and Related Conditions. Journal of Anxiety Disorders, 25(3), 456-465.

Pietrzak, R. H., Tsai, J., Armour, C., Mota, N., Harpaz-Rotem, I., \& Southwick, S. M. (2015). Functional significance of a novel 7-factor model of DSM-5 PTSD symptoms: Results from the National Health and Resilience in Veterans Study. Journal of Affective Disorders, 174, 522-526.

Rosellini, A. J., Coffey, S. F., Tracy, M., \& Galea, S. (2014). A person-centered analysis of posttraumatic stress disorder symptoms following a natural disaster: Predictors of latent class membership. Journal of Anxiety Disorders, 28(1), 16-24.

Rosen, G. M. (2004). 5 Malingering and the PTSD Data Base. Issues and Controversies, 85.

Ruscio, A. M., Ruscio, J., \& Keane, T. M. (2002). The latent structure of posttraumatic stress disorder: a taxometric investigation of reactions to extreme stress. Journal of Abnormal Psychology, 111(2), 290.

Sareen, J., Houlahan, T., Cox, B. J., \& Asmundson, G. J. (2005). Anxiety disorders associated with suicidal ideation and suicide attempts in the National Comorbidity Survey. The Journal of Nervous and Mental Disease, 193(7), 450-454. 
Schmittmann, V. D., Cramer, A. O., Waldorp, L. J., Epskamp, S., Kievit, R. A., \& Borsboom, D. (2013). Deconstructing the construct: A network perspective on psychological phenomena. New Ideas in Psychology, 31(1), 43-53.

Simms, L. J., Watson, D., \& Doebbelling, B. N. (2002). Confirmatory factor analyses of posttraumatic stress symptoms in deployed and nondeployed veterans of the Gulf War. Journal of Abnormal Psychology, 111(4), 637.

Sundin, J., Fear, N. T., Iversen, A., Rona, R. J., \& Wessely, S. (2010). PTSD after deployment to Iraq: conflicting rates, conflicting claims. Psychological Medicine, 40(03), 367-382.

Thompson, R., Henkel, V., \& Coyne, J.C. (2004). Suicidal ideation in primary care; ask a vague question, get a confusing answer. Psychosomatic Medicine, 66, 455-456

Tibshirani, R. (1996). Regression shrinkage and selection via the lasso. Journal of the Royal Statistical Society. Series B (Methodological), 267-288.

Tsai, J., Harpaz-Rotem, I., Armour, C., Southwick, S. M., Krystal, J. H., \& Pietrzak, R. H. (2014). Dimensional structure of DSM-5 posttraumatic stress disorder symptoms: Results from the National Health and Resilience in Veterans Study. The Journal of Clinical Psychiatry, 76(5), $1-478$.

van Borkulo, C. D., Borsboom, D., Epskamp, S., Blanken, T. F., Boschloo, L., Schoevers, R. A., \& Waldorp, L. J. (2014). A new method for constructing networks from binary data. Scientific Reports, 4.

Ware, J. E., Kosinski, M., Turner-Bowker, D. M., \& Gandek, B. (2002). How to score version 2 of the SF-12 health survey (with a supplement documenting version 1). QualityMetric Incorporated.

Weathers, F. W., Litz, B. T., Keane, T. M., Palmieri, P. A., Marx, B. P., \& Schnurr, P. P. (2013). The PTSD Checklist for DSM-5 (PCL-5). Scale available from the National Center for PTSD at www. ptsd.va.gov.

Weathers, F. W., Blake, D. D., Schnurr, P. P., Kaloupek, D. G., Marx, B. P., \& Keane, T. M. (2013). The Clinician-Administered PTSD Scale for DSM-5 (CAPS-5). Scale available from the National Center for PTSD at www. ptsd.va.gov. 